\title{
Digital media consumption and youth's interests in ecosystem services, sustainability and science as a means for disease prevention
}

\author{
Ibrahim Niankara, Muhammad N. Al-adwan ${ }^{\mathrm{a}, \mathrm{b}}$ \\ ${ }^{a}$ College of Business, Al Ain University of Science and Technology, P.O. Box: 112612, Abu Dhabi, UAE; Tel: \\ +97124444696 Ext: 539 ; Fax: +97124444304 ; E-mail: ibrahim.niankara@aau.ac.ae \\ ${ }^{b}$ College of Communication 85 Media, Al Ain University of Science and Technology, P.O. Box: 112612, Abu \\ Dhabi, UAE; E-mail: muhammadnoor.aladwan@aau.ac.ae
}

\begin{abstract}
Along with the advantages associated with access to information and fast communication, screen time from increased digital media consumption has recently been associated with adverse effects on youth well-being. To get a clearer picture of its value for global youth based sustainability initiatives, this study investigates the effects of increased digital media consumption on youth's interests in ecosystem services, sustainability and science as a means for disease prevention. We achieve this, using data on 187821 adolescent students from 50 countries worldwide. Methodologically, we rely on a mixed bivariate ordered probit representation of youth's joint interest in the biosphere (ecosystem services and sustainability) and science as a means for disease prevention, which we then estimate using Markov Chain Monte Carlo (MCMC) Methods.

We found that each level increase in adolescent students' reported frequency of news blogs visits and web browsing on broad science adversely affect their interests in ecosystem services, sustainability and science as a means for disease prevention. Although each level increase in youth's frequency of ecological website visits also reduces by $20 \%$ (with $95 \%$ CI $[-0.36 ;-0.32]$ ) their interests in the biosphere, it is found to increase however by $3 \%$ (with $95 \%$ CI $[0.02 ; 0.05]$ ) their interest science as a means for disease prevention. Overall, our results highlight heterogeneous effects of digital media consumption on adolescents' wellbeing in terms of their interests in ecosystem services, sustainability, and science as a means for disease prevention.
\end{abstract}

Keywords: Bayesian methods, Digital media, Ecosystem services, Sustainable development, Youth health JEL: C11, D83, I15, I18, P5, Q01 


\section{Introduction}

The year 2015 represents a key milestone in the global health and development community, with the end of the Millennium Development Goals (MDGs) era and the launch of the 17 Sustainable Development Goals (SDGs), which define 169 targets, and 230 indicators leading up to 2030 (Lim et al., 2016). The goals are categorized into five groups, the so-called 5Ps: People, Planet, Partnership, Peace, and Prosperity, which should be promoted by all countries to create a sustainable world (Nakatani, 2016). Although significant improvements have been recorded for risk factor exposure and global burden of disease (Lim et al., 2016), the transition from MDGs to SDGs shifted the global health paradigm, changing the environment surrounding health strategies and plans (Nakatani, 2016), and thereby leaving much to be done to meet the defined health and non-health related SDGs targets by 2030 (Maurice, 2016).

As the bearers of the burden of past and current decisions, young people have been identified as critical stakeholders in sustainability initiatives such as those embraced under the UN 2030 Agenda (De Leeuw et al., 2015). Globally, young people age 10-24 represent 27\% of the world's population. As an important share of the world population, they also tend to be more vulnerable and at greater risk of disease and poor health (including mental and reproductive health)(Kleinert and Horton, 2016). The latest report using the Global Burden of Disease (GBD) data suggests that changing patterns of global youth health have the potential to undermine future population health as well as global economic development, unless timely and effective strategies are put into place to revert these tendencies (Mokdad et al., 2016). In addition, as quoted by former UN secretary general (Ki-moon, 2016):

"Adolescence is a critical life stage during which individuals must have the opportunity to develop the capabilities required for realizing their full potential and achieving a prosperous, healthy, and fulfilling life. Transitions from adolescence to adulthood require investments in health information and services tailored to adolescents' needs, quality education, vocational training, and the chance to enter the productive workforce, as well as human rights protections and opportunities to participate in decision-making. Such investments will benefit not only young people who need them, but also their communities and countries". Further reiterating the importance of youth health and well-being in national and global sustainability initiatives.

Because young people are constant users of digital technology (Wartella et al. 2016) including the internet and mobile phones (Chassiakos et al., 2016; Kabali et al., 2015; Lissak, 2018), they open up to many innovative possibilities for health education and disease prevention involving these technologies (Bailey et al., 2015; Benjamin and Potts, 2018; Carreiro et al., 2018; Cowey and Potts, 2018; Kim et al., 2015; Lupton, 2013, 2014). Indeed, by affecting youth's ability to readily access, understand, and use unbiased evidence-based health information, digital media contributes to raising health awareness (Sørensen et al., 2012) and literacy (Guo et al., 2018; Trezona et al., 2018), thereby reducing exposures to risk factors, and in so doing illness (Neubeck et al., 2015), 
and overall disease burden (Nutbeam, 2000; Early and Bustillos, 2018).

Along with the above declined advantages, a growing body of literature associates excessive and addictive digital media consumption among youth, with physical Dumuid et al. (2017), psychological Babic et al. (2017), social and neurological (Twenge and Campbell, 2018) adverse consequences. Among the identified physical health effects are poor sleep and risk factors for cardiovascular diseases such as high blood pressure, obesity, low HDL cholesterol, poor stress regulation, impaired vision, and reduced bone density (Chiasson et al., 2016; Poitras et al., 2017). On the other hand, the identified psychological effects include among others mobile phone dependency, depressive symptoms and suicidal behavior due to poor sleep(Rosen et al., 2014; Twenge et al., 2018). The neurological effects on the other hand include craving behaviors, decreased social coping, and brain structural changes due to cognitive control and emotional regulation (Lissak, 2018).

Since the literature on the effect of digital media on adolescents' well-being remains divided (Bell et al., 2015; Chassiakos et al., 2016; Orben and Przybylski, 2019), the current study makes use of a unique data set(Niankara, 2019), to investigate the value of digital media for global youth based sustainability initiatives. The general question we seek to address is:

How much does digital media consumption among adolescents worldwide affect their knowledge of and interest in ecosystem services, sustainability and how science can help prevent diseases? To the best of our knowledge, this question has not been specifically addressed in the scientific discourse. Given the exploratory nature of our investigation, and the relative gravity of the type I error compared to the type II error in statistical testing and policy recommendation, we adopt the conservative view under which the true state of nature is that digital media consumption among youth has no bearing on their interest in ecosystem services, sustainability, and science as a means for disease prevention. This leads us to explicitly formulate and test the following null hypotheses:

1. $H_{O 1}$ : Frequent visits of ecological websites have no bearing on adolescents' level of interest in ecosystem services, sustainability and science as a means for disease prevention;

2. $H_{O 2}$ : Frequent visits of news blogs have no bearing on adolescents' level of interest in ecosystem services, sustainability and science as a means for disease prevention;

3. $H_{O 3}$ : Frequent web browsing on broad science have no bearing on adolescents' level of interest in ecosystem services, sustainability and science as a means for disease prevention;

4. $H_{O 4}$ : There is no relationship between adolescents' interest in the biosphere (ecosystem services and sustainability) and their interest in science as a means for disease prevention.

To formally test the above four hypotheses, we organize the rest of the paper as follows: section 2 describes our methodology, by first presenting the data, followed by the econometric framework and identification strategy; section 3 presents the findings; while section 4 discusses and concludes the analysis. 


\section{Methods}

\subsection{Data and Variables Description}

This paper uses data from the "Cross-national Data Sample on the Environmental Affection and Cognition of Adolescent Students of Varying Interests in Ecosystem Services and Sustainability" (Niankara, 2019). This data contains information on 187821 students from 50 countries worldwide, and is extracted from the student questionnaire file of the Program for International Student assessment (PISA) 2015 (OECD, 2016). PISA is the triennial survey of adolescent students around the world lunched by the Organization for Economic Co-operation and Development (OECD), to assess the extent to which the students near the end of their compulsory education, have acquired key knowledge and skills essential for full participation in modern societies. A detailed description of its sampling design is found in the OECD report(OECD, 2017, 67-91). Table (1) provides definitions and summary statistics for all the variables used in the present analysis.

\subsubsection{Dependents Variables}

The dependent variables in this study are adolescent students' level of interest in ecosystem services and sustainability (IntBiosph), and their interest level in science as a means for disease prevention (IntScPrevDis):

- IntBiosph: also defined as $y_{1}$, is a qualitative ordinal variable taking the values (1- unaware and not interested, 2-Hardly interested, 3-Aware and Interested). As shown in table (1) it has a mean value of 2.28 and a standard deviation of 0.79 .

- IntScPrevDis: defined as $y_{2}$, is also a qualitative ordinal variable taking the values (1- unaware and not interested, 2-Hardly interested, 3-Aware and Interested). And as shown in table (1) it has a mean value of 2.62 and a standard deviation of 0.68 .

\subsection{Econometric Model Specification}

Our mixed bivariate ordered probit (MBOP) model of adolescent students' interest in the biosphere and in science as a means for disease prevention, is adapted from the latent variable framework (Sajaia, 2008; Niankara and Zoungrana, 2018). For this, we assume that students' latent propensity to be interested in ecosystem services and sustainability $y_{1}^{*}$ and their propensity to be interested in science as a means for disease prevention $y_{2}^{*}$ are determined by the following system of linear mixed equations:

$$
\begin{aligned}
& y_{1 i}^{*}=X^{\prime}{ }_{1 i} \beta_{1}+Z^{\prime}{ }_{1 i} u_{1}+\epsilon_{1 i} \\
& y_{2 i}^{*}=X^{\prime}{ }_{2 i} \beta_{2}+Z^{\prime}{ }_{2 i} u_{2}+\epsilon_{2 i}
\end{aligned}
$$


Where $X^{\prime}{ }_{1 i}$, and $X^{\prime}{ }_{2 i}$ represent the fixed effects vectors of explanatory variables, and include the indicators of adolescent students' frequency of digital media consumption (EcoWebVisit, BlogsVisit, BroadScWeb), and the socio-economic and demographic control variables (AGE, Gender, GradeLev, IMMIG, MISCED, FISCED, ESCS); $Z^{\prime}{ }_{1 i}$, and $Z^{\prime}{ }_{2 i}$ represent the random effects vectors of explanatory variables, and include only (CNTRYID) in the present study. $\beta_{1}$ and $\beta_{2}$ are the corresponding vectors of unknown fixed effects, while $u_{1}$, and $u_{2}$ are the corresponding unknown random country effects. $\epsilon_{1 i}$ and $\epsilon_{2 i}$ are the error terms of the system, and $i$ denotes the individual student subscript. The explanatory variables in the model are assumed exogenous such that $E\left(X^{\prime}{ }_{1 i} \epsilon_{1 i} \mid Z_{1 i}^{\prime}\right)=E\left(X_{2 i}^{\prime} \epsilon_{2 i} \mid Z_{2 i}^{\prime}\right)=0$.

The observed indicators of adolescent students' levels of interest $y_{1 i}$, and $y_{2 i}$ are related to their corresponding latent propensities as:

$$
\begin{array}{r}
y_{1 i}= \begin{cases}1-\text { Unaware and Not interested } & \text { if } y_{1 i}^{*} \leq \mu_{1} \\
2-\text { Hardly Interested } & \text { if } \mu_{1} \leq y_{1 i}^{*} \leq \mu_{2} \\
3-\text { Aware and Interested } & \text { if } \mu_{2}<y_{1 i}^{*}\end{cases} \\
y_{2 i}= \begin{cases}1-\text { Unaware and Not interested } & \text { if } y_{2 i}^{*} \leq \delta_{1} \\
2-\text { Hardly Interested } & \text { if } \delta_{1} \leq y_{2 i}^{*} \leq \delta_{2} \\
3-\text { Aware and Interested } & \text { if } \delta_{2}<y_{2 i}^{*}\end{cases}
\end{array}
$$

Where the unknown cutoffs satisfy the condition that $\mu_{1}<\mu_{2}$ and $\delta_{1}<\delta_{2}$. For identification purposes, the first threshold values are "anchored" a priori, such that $\mu_{1}=\delta_{1}=0$. Following (Jackman, 2000; McKelvey and Zavoina, 1975), we also define $\mu_{0}=\delta_{0}=-\infty$ and $\mu_{3}=\delta_{3}=+\infty$ in order to avoid handling the boundary cases separately. The joint probability of $y_{1 i}=j$ and $y_{2 i}=k$ is:

$$
\begin{aligned}
\operatorname{Pr}\left(y_{1 i}=j, y_{2 i}=k\right) & =\operatorname{Pr}\left(\mu_{j-1}<y_{1 i}^{*} \leq \mu_{j}, \delta_{k-1}<y_{2 i}^{*} \leq \delta_{k}\right) \\
& =\operatorname{Pr}\left(y_{1 i}^{*} \leq \mu_{j}, y_{2 i}^{*} \leq \delta_{k}\right) \\
& -\operatorname{Pr}\left(y_{1 i}^{*} \leq \mu_{j-1}, y_{2 i}^{*} \leq \delta_{k}\right) \\
& -\operatorname{Pr}\left(y_{1 i}^{*} \leq \mu_{j}, y_{2 i}^{*} \leq \delta_{k-1}\right) \\
& +\operatorname{Pr}\left(y_{1 i}^{*} \leq \mu_{j-1}, y_{2 i}^{*} \leq \delta_{k-1}\right)
\end{aligned}
$$

If $\epsilon_{1 i}$ and $\epsilon_{2 i}$ are distributed as bivariate standard normal with correlation $\rho$, then each student's contribution to the likelihood function could be expressed as: 


$$
\begin{aligned}
\operatorname{Pr}\left(y_{1 i}=j, y_{2 i}=k\right) & =\Phi_{2}\left(\mu_{j}-X^{\prime}{ }_{1 i} \beta_{1}-Z^{\prime}{ }_{1 i} u_{1},\left(\delta_{k}-X^{\prime}{ }_{2 i} \beta_{2}-Z^{\prime}{ }_{2 i} u_{2}\right) \zeta, \tilde{\rho}\right) \\
& -\Phi_{2}\left(\mu_{j-1}-X^{\prime}{ }_{1 i} \beta_{1}-Z^{\prime}{ }_{1 i} u_{1},\left(\delta_{k}-X^{\prime}{ }_{2 i} \beta_{2}-Z_{2 i}{ }_{2 i} u_{2}\right) \zeta, \tilde{\rho}\right) \\
& -\Phi_{2}\left(\mu_{j}-X^{\prime}{ }_{1 i} \beta_{1}-Z^{\prime}{ }_{1 i} u_{1},\left(\delta_{k-1}-X^{\prime}{ }_{2 i} \beta_{2}-Z^{\prime}{ }_{2 i} u_{2}\right) \zeta, \tilde{\rho}\right) \\
& +\Phi_{2}\left(\mu_{j-1}-X^{\prime}{ }_{1 i} \beta_{1}-Z^{\prime}{ }_{1 i} u_{1},\left(\delta_{k-1}-X^{\prime}{ }_{2 i} \beta_{2}-Z_{2}{ }_{2 i} u_{2}\right) \zeta, \tilde{\rho}\right)
\end{aligned}
$$

where $\Phi_{2}$ is the bivariate standard normal cumulative distribution function, $\zeta=\frac{1}{\sqrt{1+2 \gamma \rho+\gamma^{2}}}$ and $\tilde{\rho}=\zeta(\gamma+\rho)$. This specification is referred to as mixed simultaneous bivariate ordered probit model, and is estimated within the R statistical software(R Core Team, 2015), using the package MCMCglmm Hadfield (2010), which we describe next.

\subsection{Model Estimation}

Because the Mixed Bivariate Odered Probit model as represented in equation (1) is just a special case of Generalized Linear Mixed Models (GLMMs) with a probit link function on each outcome variable, we adapt the Markov Chain Monte Carlo GLMMs framework by Hadfield (2010) to estimate our model. To achieve this, we stack the vectors of adolescent students' latent propensities to be interested in the biosphere (ecosystem services, sustainability) $y_{1 i}^{*}$, and in science as a means for disease prevention $y_{2 i}^{*}$, into a single column vector $Y^{*}=\left(y_{1 i}^{*}, y_{2 i}^{*}\right)$ with

$$
Y^{*}=\mathbf{X} \boldsymbol{\beta}+\mathbf{Z} \mathbf{u}+\mathbf{e}
$$

where $\mathbf{X}$ and $\mathbf{Z}$ represent respectively the design matrices relating the fixed and random explanatory variables to the latent propensities. These predictors have associated parameter vectors $\boldsymbol{\beta} \sim N\left(\boldsymbol{\beta}_{0}, \mathbf{B}\right)$, and $\mathbf{u} \sim N(\mathbf{0}, \mathbf{G})$. $\mathbf{e}$ is the vector of residuals and assumed to be distributed $\mathbf{e} \sim N(\mathbf{0}, \mathbf{R})$.

The joint probability distribution of the location effects $(\boldsymbol{\beta}$ and $\mathbf{u})$ and the residuals $(\mathbf{e})$ is therefore multivariate normal:

$$
\left[\begin{array}{c}
\boldsymbol{\beta} \\
\mathbf{u} \\
\mathbf{e}
\end{array}\right] \sim N\left(\left[\begin{array}{c}
\boldsymbol{\beta}_{0} \\
\mathbf{0} \\
\mathbf{0}
\end{array}\right],\left[\begin{array}{ccc}
\mathbf{B} & \mathbf{0} & \mathbf{0} \\
\mathbf{0} & \mathbf{G} & \mathbf{0} \\
\mathbf{0} & \mathbf{0} & \mathbf{R}
\end{array}\right]\right)
$$

where $\mathbf{B}, \mathbf{G}$ and $\mathbf{R}$, are the expected (co)variance matrices of the fixed effects, random effects and residuals respectively. These are typically unknown, and must be estimated from the data. The zero off-diagonal matrices indicate a prior independence between fixed effects, random effects, and residuals.

Typically, parameter estimation for GLMMs involves maximum likelihood (ML) or variants of ML. The solutions are usually iterative and numerically intensive. With recent advances in numerical 
methods and computing power, Bayesian MCMC methods are now providing more efficient alternatives (Rastogi and Merovci, 2018). For maximum likelihood based solutions see McCulloch and Searle (2001). Here we rely on the Bayesian approach, following Hadfield (2010). Since all effects are treated as random in a Bayesian analysis, we make no distinction between fixed and random effects. Following thereforeNiankara $(2018)$ we combine the design matrices $(\mathbf{W}=[\mathbf{X}, \mathbf{Z}])$ and also the parameters $\left(\boldsymbol{\theta}=\left[\boldsymbol{\beta}^{\prime}, \mathbf{u}^{\prime}\right]\right)$, and rewrite equation (6) as:

$$
Y^{*}=\mathbf{W} \boldsymbol{\theta}+\mathbf{e}
$$

Because of the multi-dimensionality of the choice probabilities, and also the intractability in integrating over the random effects (McCulloch and Searle, 2001), we refer to Bayesian Markov Chain Monte Carlo (MCMC) methods to estimate the parameters $\boldsymbol{\theta}$ of the model. MCMC methods provide an alternative strategy for marginalizing the random effects that may be more robust than the techniques used to approximate the integrals (Zhao et al., 2006; Brown and Draper, 2006).

\subsection{Bayesian MCMC Sampling Schemes for Identification of the Model}

Using the multivariate representation in equation (8), the prior distribution for the location effects $(\boldsymbol{\theta})$ is multivariate normal and therefore Gibbs sampled in a single block using the method of Gracia-Cortes and Sorensen (2001) as explained below. With normal conjugate priors, the variance structures ( $\mathbf{R}$ and $\mathbf{G}$ ) follow an inverse-Wishart distribution which is also Gibbs sampled in a single block. The variance structures $(\mathbf{R}$ and $\mathbf{G})$ in equation $(7)$ are represented as:

$$
\mathbf{G}=\mathbf{V}_{1} \otimes \mathbf{A}_{1}
$$

where $\mathbf{V}_{1} \otimes \mathbf{A}_{1}$ is the expected (co)variance matrix corresponding to the random effect (CNTRYID) as initially explained in the formulation of equation (1). The (co)variance matrices (V) are lowdimensional and are to be estimated, while the structured matrices (A) are high dimensional and treated as known. The Kronecker product $(\otimes)$ within component terms allows for dependence between country random effects. Controlling for the between country random variations in students interest, all remaining variations are assumed idiosyncratic, and captured by the residuals' variance structure $\mathbf{R}$.

\subsubsection{Updating the Latent propensities}

For a given adolescent student in our sample, the conditional density of the latent propensities to be interested in the biosphere (ecosystem services, sustainability), and in science as a means for disease prevention is a (2) dimensional latent vector $Y_{i}^{*}$ given by:

$$
P\left(Y_{i}^{*} \mid \mathbf{y}, \boldsymbol{\theta}, \mathbf{R}, \mathbf{G}\right) \propto f_{i}\left(y_{i} \mid Y_{i}^{*}\right) f_{N}\left(e_{i} \mid \mathbf{r}_{i} \mathbf{R}_{/ i}^{-1} \mathbf{e}_{/ i}, r_{i}-\mathbf{r}_{i} \mathbf{R}_{/ i}^{-1} \mathbf{r}_{i}^{\prime}\right)
$$


where $f_{N}$ represents the multivariate normal distribution with specified mean vector and (co)variance matrix. Hence equation (10) suggests that the conditional density of the latent propensity vector for student $i$, is proportional to the product of the conditional distribution of the observed student interest level $y_{i}$, given the latent propensity $Y_{i}^{*}$ and the probability density of the propensity residuals. The 2 dimensional vector of latent propensity residuals $e_{i}$ for student $i$ follows a conditional normal distribution, where the conditioning is on the $2 \times(N-1)$ residuals associated with the other students in the sample. The notation $/ i$ denotes vectors or matrices with the $i^{\text {th }}$ row and or column removed. This conditioning accounts for residual correlation across students.

Since latent propensities are updated in blocks of correlated residuals, we control for the correlations between and within students. This is represented using the block notation, where a block is a group of residuals expected to be correlated in equation (8). Equation (10) can then be rewritten as:

$$
P\left(y_{k}^{*} \mid \mathbf{y}, \boldsymbol{\theta}, \mathbf{R}, \mathbf{G}\right) \propto p_{i}\left(y_{i} \mid y_{k}^{*}\right) f_{N}\left(\mathbf{e}_{k} \mid \mathbf{0}, \mathbf{R}_{k}\right)
$$

where $k$ indexes blocks of latent propensities in equation (8), that have non-zero residual covariances. Since residuals are assumed to be correlated across the two latent propensities for each student, and also across students, we have a total of $(N) \times(2)$ residual correlations, when $k=1$. As such, the conditional density of each latent propensity $y_{i j}^{*}$ for all $i=1 \cdots N$ and $j=1 \cdots 2$ is obtained by conditioning each $e_{i j}$ on the remaining $1+(N-1) \times 2$ residuals.

The average posterior (co)variance matrix $\mathbf{M}$ of the single $2 \times N$ dimensional block vector $y_{k}^{*}$ with $k=1$ is updated at each iteration of the burn-in period following Haario et al. (2001). An efficient multivariate proposal density with covariance matrix $\nu \mathbf{M}$ is determined using adaptive methods during the burn-in phase. The scalar $\nu$ is obtained using the method of Ovaskainen et al. (2008) so that the proportion of successful jumps in the Markov Chain is optimal at a rate of 0.23 for the multidimensional vector $y_{k}^{*}$ with $k=1$ (Gelman et al., 2004).

\subsubsection{Updating the location effects vector}

The location effects vector $\left(\boldsymbol{\theta}=\left[\boldsymbol{\beta}^{\prime}, \mathbf{u}^{\prime}\right]\right)$ is sampled as a block using a method by Gracia-Cortes and Sorensen (2001) which involves solving the sparse linear system:

$$
\tilde{\boldsymbol{\theta}}=\mathbf{C}^{-1} \mathbf{W}^{\prime} \mathbf{R}^{-1}\left(\mathbf{l}-\mathbf{W} \boldsymbol{\theta}_{\star}-\mathbf{e}_{\star}\right)
$$

This system is solved using cholesky factorization from the Csparse library in R by Davis (2006). $\mathbf{C}$ is a sparse matrix (populated primarily with zeros) representing the model coefficient matrix:

$$
\mathbf{C}=\mathbf{W}^{\prime} \mathbf{R}^{-1} \mathbf{W}+\left[\begin{array}{cc}
\mathbf{B}^{-1} & \mathbf{0} \\
\mathbf{0} & \mathbf{G}^{-1}
\end{array}\right]
$$

$\boldsymbol{\theta}_{\star}=\left[\boldsymbol{\beta}_{\star}^{\prime}, \mathbf{u}_{\star}^{\prime}\right]$ and $\mathbf{e}_{\star}$ are random draws from the multivariate normal distributions:

$$
\left[\begin{array}{l}
\boldsymbol{\beta}_{\star} \\
\mathbf{u}_{\star}
\end{array}\right] \sim N\left(\left[\begin{array}{c}
\boldsymbol{\beta}_{0} \\
\mathbf{0}
\end{array}\right],\left[\begin{array}{cc}
\mathbf{B} & \mathbf{0} \\
\mathbf{0} & \mathbf{G}
\end{array}\right]\right)
$$


and

$$
\mathbf{e}_{\star} \sim N\left(\mathbf{W} \boldsymbol{\theta}_{\star}, \mathbf{R}\right)
$$

A realization from the required probability distribution $P\left(\boldsymbol{\theta} \mid Y^{*}, \mathbf{W}, \mathbf{R}, \mathbf{G}\right)$ is then obtained as $\tilde{\boldsymbol{\theta}}+\boldsymbol{\theta}_{\star}$

\subsubsection{Updating the variance structures $G$ and $R$}

All information for the estimation of the variance structures $\mathbf{G}$ and $\mathbf{R}$ comes from the inverseWishart prior distribution, following the conditional sampling strategy described in Korsgaard et al. (1999). For the $\mathbf{G}$ structure as represented in equation (9), the sum of squares matrix associated with the single random effect component has the form:

$$
\mathbf{S}=\phi^{\prime} \mathbf{A}^{-1} \boldsymbol{\phi}
$$

where $\phi$ is a matrix of random effects with each row indexing the relevant row/column of $\mathbf{A}$, and each column indexing the relevant row/column in $\mathbf{V}$, and also $\mathbf{A}$ and $\mathbf{V}$ defined as in equation (9). The parameter (co)variance matrix can then be sampled from the inverse-Wishart distribution:

$$
\mathbf{V} \sim \mathbf{I W}\left(\left(\mathbf{S}_{p}+\mathbf{S}\right)^{-1}, n_{p}+n\right)
$$

where $\mathbf{S}_{p}$ and $n_{p}$ are the prior sum of squares and prior degree's of freedom, respectively, and $n$ is the number of rows in the matrix of random effects $\phi$.

\subsubsection{Updating the Cutoff Points}

The methods developed by (Cowles, 1996), are used to allow the latent propensities and cutoff points to be updated simultaneously using a Hastings-with-Gibbs update.

\section{Findings}

The results of the analysis are divided into two parts; the first part covers univariate and bivariate descriptive statistics, while the second part covers the econometric results from the Bayesian Markov Chain Monte Carlo estimation of the MBOP model.

\subsection{Descriptive Results}

The descriptive results in table (1) suggest that $48 \%$ of adolescent student respondents are males, with an average age of 15.79 years. The mean value of the standard normalized scale 
of the index of economic, social and cultural status (ESCS) 1 , shows that the average adolescent student in the sample is 0.04 standard deviation below the mean index value across all PISA 2015 students. On average students have completed grade 9 (3.70) and are aware(2.28) of the biosphere (ecosystem services and sustainability), and also interested (2.62) in how science can help prevent diseases. Table (1) also shows that on average adolescent students regularly visit ecological websites (3.40), news blogs (3.28) and websites on broad science (3.06). Furthermore both parents have on average at least a post-secondary non-tertiary education, mother (4.24) and father (4.20), based the UNESCO international standard classification of education.

In order to understand the unconditional relationships between adolescent students' frequency of digital media consumption and their interests in the biosphere (IntBioshp) and in science as a means for disease prevention (IntScPrevDis), we perform a series of chi-square tests between each of the three digital media types, and the two ordinal outcome measures. The results of these tests are presented along with the corresponding conditional and unconditional frequency distributions in tables (2) and (3) respectively for "IntBioshp" and "IntScPrevDis". As shown in table (2) each of the three measures of digital media consumptions (EcoWebVisit, BlogsVisit, and BroadScWeb) are significantly associated with adolescent students' interest in ecosystem services and sustainability (IntBioshp) since the corresponding p-values for these tests are all less than the $5 \%$ significance level. Table (2) also shows that the relative frequency of adolescent students increases with increasing levels of digital media consumption. This observation is true for all three measures of digital media consumption- EcoWebVisit (4.5\%, 8.9\%, 28.5\%, 58.1\%), BlogsVisit ( $6.5 \%, 11.8 \%, 29.3 \%, 52.5 \%)$, and BroadScWeb (7.6\%, 15.5\%, 39.9\%, 37\%). Similarly, the last row of table (2) shows that the relative frequency of adolescent students increases with increasing levels of students interest in ecosystem services and sustainability $(21.2 \%, 29.6 \%$, and $49.2 \%)$. The same observations made above with respect to the chi-square tests results, and relative frequency distribution patterns hold true for adolescent students interest in science as a means for disease prevention, which can be seen in table (3).

\subsection{Econometric Results}

For the MCMC estimation, we defined 50000 iterations of the algorithm with a burn-in period of 15000 iterations, and a thinning interval of 10 iterations. This has led to an effective random sample of 3500 Markov draws, which were then used to estimate the parameters of the model, along with their 95\% confidence intervals (CI). Presentation of these econometric results is divided

\footnotetext{
${ }^{1}$ Estimated across all OECD countries and partner countries on the basis of the following variables: the International Socio-Economic Index of Occupational Status (ISEI); the highest level of education of the student's parents in years of schooling; the PISA index of family wealth; the PISA index of home educational resources; and the PISA index of possessions related to "classical" culture in the family home. See (OECD, 2017, pp. 339-340) for more details
} 
into five sub-sections; the first subsection shows the interval and point estimates for the random effects, residuals and cutoff-points in the MBOP model. The second subsection focuses on describing the effects of digital media consumption on adolescent students' interest in the biosphere (ecosystem services and sustainability). The third sub-section describes the effects of digital media consumption on adolescent students' interest in science as a means for disease prevention. The fourth sub-section focuses on describing the effects of the control variables on their interests in the biosphere, while the fifth sub-section focuses on describing the effects of the control variables on their interests in science as a means for disease prevention.

\subsubsection{Random effects, residuals and cutoff points estimates}

The results of the random country variations in adolescent students interests in the biosphere (IntBiosph) and science as a means for disease prevention (IntScPrevDis) are summarized in table (4). Since the $95 \%$ confidence intervals on the estimated posterior mean values 0.41 (with CI[0.20 ; 0.69]) , and 0.15 (CI[0.09; 0.23]) are void of zero, we conclude their statistical significance at the $5 \%$ level. These results indicate significant country level heterogeneity in adolescent students' interests. Accounting for this source of variation, all remaining variations in adolescent students' interests in the biosphere and science as a means for disease prevention are assumed idiosyncratic, and described by the residuals' variance-covariance matrix as summarized in table (5).

The results of the estimated residuals' variance-covariance matrix in table (5) shows a variance of 2.09 (with 95\% CI $[1.84 ; 2.40]$ ) for adolescent students' interest in the biosphere (ecosystem and sustainability), and a variance of 1.58 (with $95 \%$ CI $[1.31 ; 1.81]$ ) for their interest in how science can help prevent diseases. similarly table (5) shows a positive and significant covariance value of 1.52 (with 95\% CI $[1.45 ; 1.62]$ ) between adolescent students' interest in the biosphere (IntBiosph), and their interest in how science can help prevent diseases (IntScPrevDis). This latter result suggests that the more interested adolescent students are in ecosystem services and sustainability, the more interested they also are in science as a means for disease prevention. Using the estimated covariance value, along with the two estimated variances, we calculate the correlation coefficient between "IntBiosph" and "IntScPrevDis" to be $\hat{\rho}=0.8353[1.52 /(\sqrt{2.09} * \sqrt{1.58})]$, which indicates a fairly strong positive linear relationship between the two outcome variables. The MCMC estimates for the cutoff points $\mu_{2}$ and $\delta_{2}$ as discussed in equation (3), are summarized in table (6), and show that $\hat{\mu_{2}}=1.56($ with $95 \% C I[1.50 ; 1.64])$ while $\hat{\delta_{2}}=1.02($ with $95 \% C I[0.97 ; 1.07])$.

\subsubsection{Digital media consumption and students interest in the biosphere}

The results of the effect of digital media consumption on adolescent students' interest in ecosystem services and sustainability are summarized in the first two columns of table (6), and suggest that digital media consumption negatively affects adolescent students' interest in the biosphere (ecosystem services and sustainability). Indeed all three measures of adolescent students frequency of digital media usage (EcoWebVisit, BlogsVisit, BroadScWeb) show respectively negative signs 
$(-0.2 ;-0.21 ;$ and -0.34$)$. These results indicate that each level increase in adolescent students' reported frequency of ecological websites visit is associated with a $20 \%$ decrease in their interest in ecosystem services and sustainability (with $95 \%$ CI $[-0.22 ;-0.18]$ ). This figure is $-21 \%$ (with $95 \%$ CI $[-0.23 ;-0.19]$ ) for each level increase in their reported frequency of news following via blogs; and -34\% (with $95 \%$ CI $[-0.36$; -0.32$]$ ) for each level increase in their reported frequency of web-browsing on broad science.

\subsubsection{Digital media consumption and students interest in science for disease prevention}

The results of the effect of digital media consumption on adolescent students' interest in how science can help prevent diseases are summarized in the last two columns of table (6), and show mixed results. In fact each level increase in adolescent students' reported frequency of ecological website visits is associated with a $3 \%$ increase (with $95 \%$ CI $[0.02 ; 0.05]$ ) in their interest in science as a means for disease prevention. On the opposite, each level increase in their reported frequency of news blogs following is found to reduce by $11 \%$ their interest in science as a means for disease prevention (with $95 \%$ CI $[-0.12 ;-0.09]$ ). The same negative effect is observed for each level increase in adolescent students' reported frequency of websites visits on broad science, which now accounts for a $26 \%$ decrease (with $95 \%$ CI $[-0.28 ;-0.24]$ ) in their interest in science as a means for disease prevention.

\subsubsection{Control variables and students interests in the biosphere, and science for disease prevention}

The results of the effects of the control variables on adolescent students' joint interests in the biosphere, and science as a means for disease prevention are also summarized in table (6). These results show that each year increase in adolescent students' age positively impact both, their interest in ecosystem services and sustainability by $20 \%$ (with $95 \%$ CI $[0.18 ; 0.22]$ ), but also their interests in how science can help prevent diseases by $18 \%$ (with $95 \%$ CI $[0.16 ; 0.19]$ ). Similarly, each increase in adolescent students' grade level is found to increase both their interest in the biosphere, and in how science can help prevent diseases by $13 \%$ (with $95 \%$ CI $[0.11 ; 0.15]$ ). Furthermore, each level increase in fathers' education is found to raise by $1 \%$ (with $95 \%$ CI $[0.001$; $0.02]$ ) both, adolescent students' interest in the biosphere, and their interest in science as a means for disease prevention. Conversely however, each level increase in mother's education is found to negatively affect adolescent students' interest in the biosphere -3\% (with 95\% CI [ -0.10; -0.02]) and in science as a means for disease prevention -20\% (with 95\% CI [-0.03; -0.01]). The coefficient estimates for gender suggests that compared to female adolescent students, males show $16 \%$ less interest in ecosystem services and sustainability (with $95 \%$ CI [ $-0.19 ;-0.15]$ ), and $53 \%$ less interest in how science can help prevent diseases (with $95 \%$ CI $[-0.59 ;-0.49]$ ). Now turning to the estimated effects of immigration status, table (6) shows that compared to native students, first generation immigrant/expatriate students have $6 \%$ less interest in ecosystem services and sustainability (with $95 \%$ CI [-0.10; -0.02]), while having $16 \%$ more interest in how science can help prevent diseases 
(with $95 \%$ CI $[0.11 ; 0.20]$ ). Conversely however, compared to native students, second generation immigrant students have respectively $9 \%$ (with $95 \%$ CI $[0.05 ; 0.13]$ ) and $17 \%$ (with $95 \%$ CI [0.13 ; 0.21]) more interest in the biosphere, and in science as a means for disease prevention. Finally a one standard deviation increase in adolescent students' normalized index of socio-economic, social, and cultural status is found to increase their interest in the biosphere by $16 \%$ (with $95 \%$ CI [ 0.14 ; 0.17]), and their interest in how science can help prevent diseases by $14 \%$ (with $95 \%$ CI [0.12; $0.16])$.

\section{Conclusions and Discussions}

The literature on the effect of digital media on adolescents' well-being remains divided(Bell et al., 2015; Orben and Przybylski, 2019). Beyond all controversies however, the potential of digital media to galvanize process efficiency in both public (Alawadhi and Scholl, 2016; Kumar, 2015; Salahuddin et al., 2016) and private (Hack and Berg, 2014; Przychodzen et al., 2018; Sun et al., 2018) sectors seems important (Yonker et al., 2015; Wartella et al., 2016). Therefore to get a clearer picture of the value of digital media for global youth based sustainability initiatives, this research looked at how digital media usage among the world youth population affects their interests in ecosystem services, sustainability and science as a means for disease prevention.

The observed statistically significant coefficient values on adolescent students' interests in ecosystem services and sustainability (IntBiosph) obtained from their increased frequency of ecological website visits, news blogs visits, and web-browsing on broad science, provide us with sufficient evidence to reject the first three hypotheses $\left(H_{O 1}, H_{O 2}, H_{O 3}\right)$. We therefore conclude that the evidence is enough to suggest that increased frequency of ecological website visits, news blogs visits, and web-browsing on broad science by the world youth significantly affect their interest in ecosystem services and sustainability. However these effects appear negative suggesting a reduction rather than an increase in youth interests. In the case of adolescent students' interest in science as a means for disease prevention, we also found that increased frequencies of news blogs visits and web-browsing on broad science significantly but negatively influence adolescent students' interests. In this latter case however, increased frequency of ecological website visits is found to positively affect youth's interest in science as a means for disease prevention.

Together, these results seem to indicate that in their current state of use, digital media in the form of news blogs and broad science web-contents are not successful at galvanizing the interest of the world youth population in ecosystem services, sustainability and science as a means for disease prevention. However, the situation is mixed for ecological websites, which as digital media also appear unsuccessful at nurturing youth's interest in ecosystem services and sustainability, but do successfully nurture their interest in science as a means for disease prevention. Although these findings align with the results of previous authors including Jelenchick and Christakis (2014); Sadhir et al. (2016); Cheever et al. (2018), they also come as a contrast to the findings in Yonker 
et al. (2015); Wartella et al. (2016), which report digital media technologies to offer exciting new means for engaging and communicating with adolescents and young adults, for the purposes of providing appropriate intervention and education.

In addition to the above discussed results, our analysis also showed a significantly strong and positive correlation of 0.835 between adolescent students' interest in the biosphere (ecosystem services and sustainability) and their interest in science as a means for disease prevention. Therefore we reject the fourth hypothesis $\left(H_{O 4}\right)$ and conclude in line with Ki-moon (2016), that a global investment aimed at raising the world adolescent population's interest in science as a means for disease prevention would significantly impact their interest in ecosystem services and sustainability, and thereby help nations move closer to their 2030 sustainable development targets (De Leeuw et al., 2015).

Overall, consistent with Chassiakos et al. (2016) our study show that digital media has heterogeneous effects on youth's interest, depending on the digital media type under consideration. This is further consistent with the divisions observed in the literature over the effect of digital media on adolescent's well-being (Bell et al., 2015; Orben and Przybylski, 2019). Although the literature has mainly focused on digital media effects on youth physical (Dumuid et al., 2017; Chiasson et al., 2016; Poitras et al., 2017; Rosen et al., 2014; Twenge et al., 2018), psychological (Babic et al., 2017), social and neurological (Twenge and Campbell, 2018; Lissak, 2018) well-being with inconsistent results, our study of its effect on youth's interest in ecosystem services, sustainability and science as a means for disease prevention is just as equally important. Mainly because increased interest will lead to more commitment, and early commitment to sustainability and science as a means for disease prevention by the younger generation is key to the present and future developments in those fields, and thereby the well-being of humanity (Council et al., 2013; Smith, 1999).

To further improve the contribution of digital media to this end, and reverse the current tendencies of adverse digital media effects, a worldwide improvement in our understanding of the value of digital media and its usage might be useful (Hirsh-Pasek et al., 2015). One way to achieve this could be through Digital Education for Sustainable Development (DESD), which could be formalized in several ways, so that each DESD program reflects the unique environmental, social and economic conditions of its locality. In doing so, it could encourage changes in behavior that will create a more sustainable future in terms of environmental integrity, economic viability, and a just society for present and future generations. Having said so, it is equally important to reiterate the retrospective cross-sectional nature of our study, therefore a prospective study relying on primary and/or longitudinal data on adolescents' blogs visits, and web-browsing for information directly related to health promotion, disease prevention and sustainability, would be a valuable future endeavor to consider, as a way of confirming our present findings. 


\section{References}

Alawadhi, Suha and Hans J Scholl (2016), Smart governance: A cross-case analysis of smart city initiatives, in 'System Sciences (HICSS), 2016 49th Hawaii International Conference on', IEEE, pp. 2953-2963.

Babic, Mark J, Jordan J Smith, Philip J Morgan, Narelle Eather, Ronald C Plotnikoff and David R Lubans (2017), 'Longitudinal associations between changes in screen-time and mental health outcomes in adolescents', Mental Health and Physical Activity 12, 124-131.

Bailey, Julia, Sue Mann, Sonali Wayal, Rachael Hunter, Caroline Free, Charles Abraham and Elizabeth Murray (2015), 'Sexual health promotion for young people delivered via digital media: a scoping review'.

Bell, Vaughan, Dorothy VM Bishop and Andrew K Przybylski (2015), 'The debate over digital technology and young people', BMJ: British Medical Journal (Online) 351, doi: https://doi.org/10.1136/bmj.h3064.

Benjamin, Katherine and Henry WW Potts (2018), 'Digital transformation in government: Lessons for digital health?'.

Brown, W. and D. Draper (2006), 'A comparison of bayesian and likelihood-based methods for fitting multilevel methods', Bayesian Analysis 1(3), 473-514.

Carreiro, Stephanie, Peter R Chai, Jennifer Carey, Jeffrey Lai, David Smelson and Edward W Boyer (2018), 'mhealth for the detection and intervention in adolescent and young adult substance use disorder', Current Addiction Reports 5(2), 110-119.

Chassiakos, Yolanda Linda Reid, Jenny Radesky, Dimitri Christakis, Megan A Moreno, Corinn Cross et al. (2016), 'Children and adolescents and digital media', Pediatrics 138(5), e20162593.

Cheever, Nancy A, Megan A Moreno and Larry D Rosen (2018), When does internet and smartphone use become a problem?, in 'Technology and Adolescent Mental Health', Springer, pp. 121131.

Chiasson, MA, R Scheinmann, D Hartel, N McLeod, J Sekhobo, LS Edmunds and S Findley (2016), 'Predictors of obesity in a cohort of children enrolled in wic as infants and retained to 3 years of age', Journal of community health 41(1), 127-133.

Council, National Research et al. (2013), Education for life and work: Developing transferable knowledge and skills in the 21st century, National Academies Press. 
Cowey, Aasha E and Henry WW Potts (2018), 'What can we learn from second generation digital natives? a qualitative study of undergraduates' views of digital health at one london university', Digital health 4, 2055207618788156.

Cowles, Mary Kathryn (1996), 'Accelerating monte carlo markov chain convergence for cumulativelink generalized linear models', Statistics and Computing 6(2), 101-111.

Davis, A. Timothy (2006), Direct Methods for Sparse Linear Systems, Fundamentals of Algoritms. SIAM, Philadelphia.

De Leeuw, Astrid, Pierre Valois, Icek Ajzen and Peter Schmidt (2015), 'Using the theory of planned behavior to identify key beliefs underlying pro-environmental behavior in high-school students: Implications for educational interventions', Journal of Environmental Psychology 42, 128-138.

Dumuid, Dorothea, Timothy Olds, Lucy K Lewis, Josep Antoni Martin-Fernández, Peter T Katzmarzyk, Tiago Barreira, Stephanie T Broyles, Jean-Philippe Chaput, Mikael Fogelholm, Gang $\mathrm{Hu}$ et al. (2017), 'Health-related quality of life and lifestyle behavior clusters in school-aged children from 12 countries', The Journal of pediatrics 183, 178-183.

Early, Jody and Dan Bustillos (2018), 'An internet for some threatens health for all: What effects could the repeal of net neutrality in the usa have on individual and population health?', Global health promotion p. 1757975918785354.

Gelman, A., J. B. Carlin, H. H. Stern and D. B. Rubin (2004), Bayesian Data Analysis, Chapman \& Hall, 2nd edition.

Gracia-Cortes, L. A. and D. Sorensen (2001), 'Alternative implementations of monte carlo em algorithms for likelihood inferences', Genetics Selection Evolution 33(4), 443-452.

Guo, Shuaijun, Elise Davis, Xiaoming Yu, Lucio Naccarella, Rebecca Armstrong, Thomas Abel, Geoffrey Browne and Yanqin Shi (2018), 'Measuring functional, interactive and critical health literacy of chinese secondary school students: reliable, valid and feasible?', Global health promotion p. 1757975918764109.

Haario, H., E. Saksman and J. Tamminen (2001), 'An adaptive metropolis algorithm', Bernoulli $7(2), 223-242$.

Hack, Stefan and Christian Berg (2014), 'The potential of it for corporate sustainability', Sustainability 6(7), 4163-4180.

Hadfield, Jarrod D. (2010), 'Mcmc methods for multi-response generalized linear mixed models: The mcmcglmm r package', Journal of Statistical Software 33(2), 1-22. 
Hirsh-Pasek, Kathy, Jennifer M Zosh, Roberta Michnick Golinkoff, James H Gray, Michael B Robb and Jordy Kaufman (2015), 'Putting education in "educational" apps: Lessons from the science of learning', Psychological Science in the Public Interest 16(1), 3-34.

Jackman, S (2000), 'Models for ordered outcomes [pdf document]', Retrieved on 25 March 2018 from Lecture notes Online Web site: http://www. stanford. edu/class/polisci203/ordered. pdf .

Jelenchick, Lauren A and Dimitri A Christakis (2014), 'Problematic internet use during adolescence and young adulthood', Adolescent Medicine: State of the Art Reviews 25(3), 605-620.

Kabali, Hilda K, Matilde M Irigoyen, Rosemary Nunez-Davis, Jennifer G Budacki, Sweta H Mohanty, Kristin P Leister and Robert L Bonner (2015), 'Exposure and use of mobile media devices by young children', Pediatrics pp. peds-2015.

Ki-moon, Ban (2016), 'Sustainability-engaging future generations now', The Lancet 387(10036), 2356-2358.

Kim, Katherine K, Holly C Logan, Edmund Young and Christina M Sabee (2015), 'Youth-centered design and usage results of the in touch mobile self-management program for overweight/obesity', Personal and Ubiquitous Computing 19(1), 59-68.

Kleinert, Sabine and Richard Horton (2016), 'Adolescent health and wellbeing: a key to a sustainable future', The Lancet 387(10036), 2355-2356.

Korsgaard, I. R., A. H. Andersen and D. Soresen (1999), 'A useful reparametrisation to obtain samples from conditional inverse wishart distributions', Genetics Selction Evolution 31(2), 177181.

Kumar, TM Vinod (2015), E-governance for smart cities, in 'E-governance for smart cities', Springer, pp. 1-43.

Lim, Stephen S, Kate Allen, Zulfiqar A Bhutta, Lalit Dandona, Mohammad H Forouzanfar, Nancy Fullman, Peter W Gething, Ellen M Goldberg, Simon I Hay, Mollie Holmberg et al. (2016), 'Measuring the health-related sustainable development goals in 188 countries: a baseline analysis from the global burden of disease study 2015', The Lancet 388(10053), 1813-1850.

Lissak, Gadi (2018), 'Adverse physiological and psychological effects of screen time on children and adolescents: Literature review and case study', Environmental research 164, 149-157.

Lupton, Deborah (2013), 'The digitally engaged patient: Self-monitoring and self-care in the digital health era', Social Theory \& Health 11(3), 256-270. 
Lupton, Deborah (2014), 'Health promotion in the digital era: a critical commentary', Health promotion international 30(1), 174-183.

Maurice, John (2016), 'Measuring progress towards the sdgs - a new vital science', The Lancet 388(10053), 1455-1458.

McCulloch, Charles E. and Shayle R. Searle (2001), Generalized Linear, and Mixed Models, New York.

McKelvey, Richard D and William Zavoina (1975), 'A statistical model for the analysis of ordinal level dependent variables', Journal of mathematical sociology 4(1), 103-120.

Mokdad, Ali H, Mohammad Hossein Forouzanfar, Farah Daoud, Arwa A Mokdad, Charbel El Bcheraoui, Maziar Moradi-Lakeh, Hmwe Hmwe Kyu, Ryan M Barber, Joseph Wagner, Kelly Cercy et al. (2016), 'Global burden of diseases, injuries, and risk factors for young people's health during 1990-2013: a systematic analysis for the global burden of disease study 2013', The Lancet 387(10036), 2383-2401.

Nakatani, Hiroki (2016), 'Global strategies for the prevention and control of infectious diseases and non-communicable diseases', Journal of epidemiology 26(4), 171-178.

Neubeck, Lis, Nicole Lowres, Emelia J Benjamin, S Ben Freedman, Genevieve Coorey and Julie Redfern (2015), 'The mobile revolution - using smartphone apps to prevent cardiovascular disease', Nature Reviews Cardiology 12(6), 350.

Niankara, Ibrahim (2018), 'Evaluating health consumers' preferences stability through joint estimation of revealed and stated health insurance preferences data', International Journal of Economics and Business Research 15(2), 236-256.

Niankara, Ibrahim (2019), 'Cross-national data sample on the environmental affection and cognition of adolescent students of varying interests in ecosystem services and sustainability', Data in Brief 22(February), 312-318.

Niankara, Ibrahim and Didier T Zoungrana (2018), 'Interest in the biosphere and students environmental awareness and optimism: A global perspective', Global Ecology and Conservation 16, e00489, https://doi.org/10.1016/j.gecco.2018.e00489.

Nutbeam, Don (2000), 'Health literacy as a public health goal: a challenge for contemporary health education and communication strategies into the 21st century', Health promotion international 15(3), 259-267. 
OECD (2016), Programme for International Student Assessment (PISA) 2015 Database: Stduent Questionnaire data file, Organization for Economic Co-operation and Development, Paris, France. Retrieved on 30 March 2018 from.

URL: http://www.oecd.org/pisa/data/2015database/

OECD (2017), PISA 2015 Technical Report: Chapter 04 - Sample design, Organization for Economic Co-operation and Development, Paris, France. Retrieved on 30 March 2018 from.

URL: http://www.oecd.org/pisa/sitedocument/PISA-2015-technical-report-final.pdf

Orben, Amy and Andrew K Przybylski (2019), 'The association between adolescent well-being and digital technology use', Nature Human Behaviour pp. DOI: https://www.nature.com/articles/s41562-018-0506-1.

Ovaskainen, O., H. Rekola, E. Meyke and E. Arjas (2008), 'Bayesian methods for analyzing movements in heterogeneous landscapes from mark-recapture data', Ecology 89(2), 542-554.

Poitras, Veronica J, Casey E Gray, Xanne Janssen, Salome Aubert, Valerie Carson, Guy Faulkner, Gary S Goldfield, John J Reilly, Margaret Sampson and Mark S Tremblay (2017), 'Systematic review of the relationships between sedentary behaviour and health indicators in the early years (0-4 years)', BMC Public Health 17(5), 868.

Przychodzen, Wojciech, Fernando Gómez-Bezares and Justyna Przychodzen (2018), 'Green information technologies practices and financial performance-the empirical evidence from german publicly traded companies', Journal of Cleaner Production 201, 570-579.

R Core Team (2015), R: A Language and Environment for Statistical Computing, R Foundation for Statistical Computing, Vienna, Austria.

URL: https://www.R-project.org/

Rastogi, Manoj Kumar and Faton Merovci (2018), 'Bayesian estimation for parameters and reliability characteristic of the weibull rayleigh distribution', Journal of King Saud University-Science $30(4), 472-478$.

Rosen, Larry D, AF Lim, Julie Felt, L Mark Carrier, Nancy A Cheever, Jose M Lara-Ruiz, Jessica S Mendoza and Jeffrey Rokkum (2014), 'Media and technology use predicts ill-being among children, preteens and teenagers independent of the negative health impacts of exercise and eating habits', Computers in human behavior 35, 364-375.

Sadhir, Mandakini, Stephanie J Stockburger and Hatim A Omar (2016), 'Challenges of internet and social media use in adolescents', Dynamics of Human Health 3(1). 
Sajaia, Zurab (2008), 'Maximum likelihood estimation of a bivariate ordered probit model: implementation and monte carlo simulations', The Stata Journal 4(2), 1-18.

Salahuddin, Mohammad, Khorshed Alam and Ilhan Ozturk (2016), 'Is rapid growth in internet usage environmentally sustainable for australia? an empirical investigation', Environmental Science and Pollution Research 23(5), 4700-4713.

Smith, Elizabeth S (1999), 'The effects of investments in thesocial capital of youth on political and civic behavior in young adulthood: A longitudinal analysis', Political psychology 20(3), 553-580.

Sørensen, Kristine, Stephan Van den Broucke, James Fullam, Gerardine Doyle, Jürgen Pelikan, Zofia Slonska and Helmut Brand (2012), 'Health literacy and public health: a systematic review and integration of definitions and models', BMC public health 12(1), 80.

Sun, Qiao, Chang Wang, Lyu-shui Zuo and Feng-hua Lu (2018), 'Digital empowerment in a weee collection business ecosystem: A comparative study of two typical cases in china', Journal of Cleaner Production 184, 414-422.

Trezona, Anita, Sarity Dodson, Paulina Mech and Richard H Osborne (2018), 'Development and testing of a framework for analysing health literacy in public policy documents', Global health promotion p. 1757975918769616.

Twenge, Jean M, Thomas E Joiner, Megan L Rogers and Gabrielle N Martin (2018), 'Increases in depressive symptoms, suicide-related outcomes, and suicide rates among us adolescents after 2010 and links to increased new media screen time', Clinical Psychological Science 6(1), 3-17.

Twenge, Jean M and W Keith Campbell (2018), 'Associations between screen time and lower psychological well-being among children and adolescents: Evidence from a population-based study', Preventive medicine reports 12, 271-283.

Wartella, Ellen, Vicky Rideout, Heather Montague, Leanne Beaudoin-Ryan and Alexis Lauricella (2016), 'Teens, health and technology: A national survey', Media and communication 4(3), 1323.

Yonker, Lael M, Shiyi Zan, Christina V Scirica, Kamal Jethwani and T Bernard Kinane (2015), " "friending" teens: systematic review of social media in adolescent and young adult health care', Journal of medical Internet research $\mathbf{1 7}(1)$.

Zhao, Y., J. Staudenmayer, B. A. Coull and M. P. Wand (2006), 'General design bayesian generalized linear mixed models', Statistical Science 21(1), 35-51. 
Table 1: Summary Description of the Variables used in the Econometric Modeling

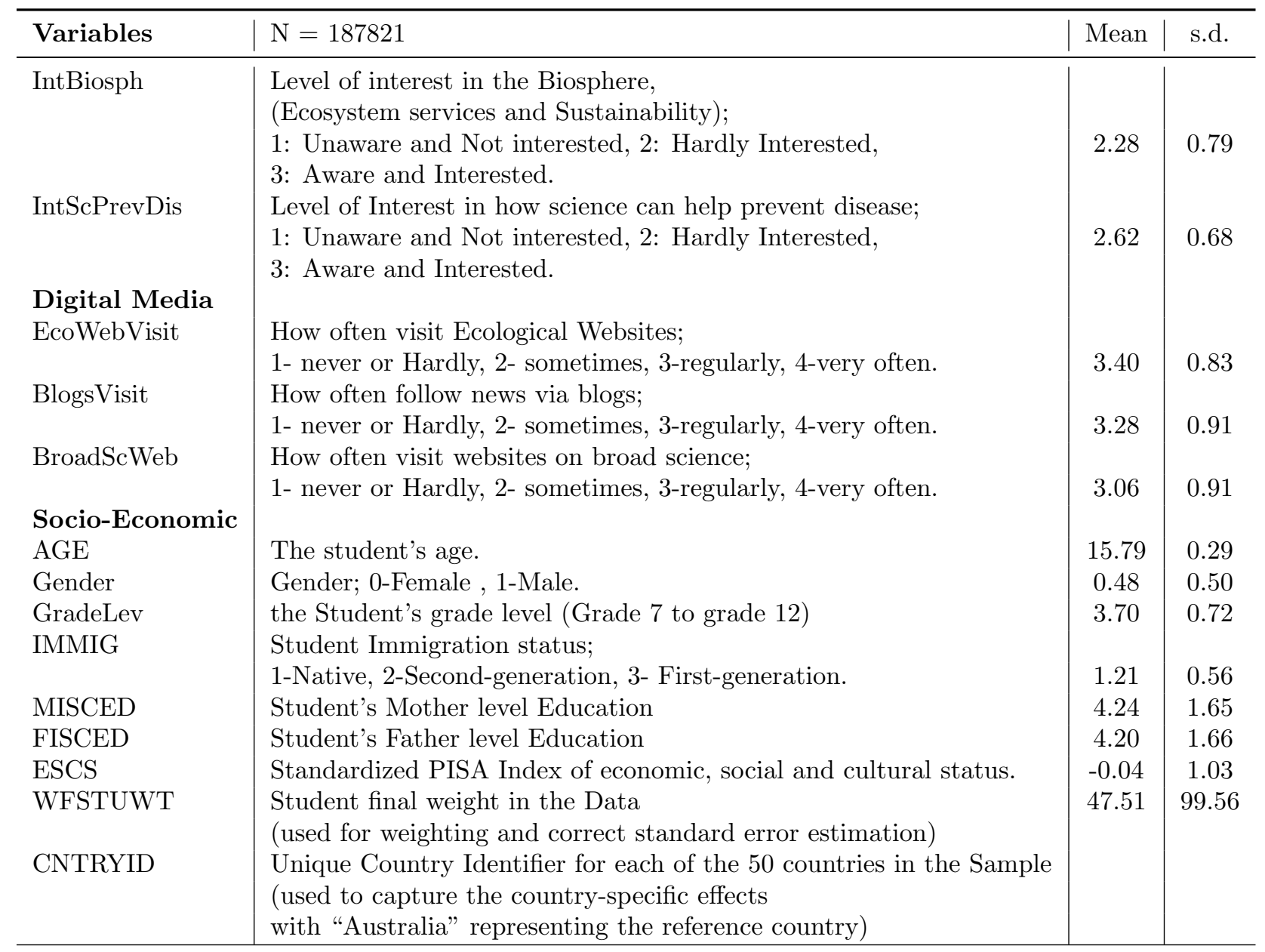

Source: Programme for International Student Assessment (PISA 2015) Data set. 
Table 2: Chi-square test results with conditional and relative frequency distributions in \% for "IntBioshp"

\begin{tabular}{|c|c|c|c|c|c|c|c|c|}
\hline & & \multicolumn{3}{|c|}{ IntBioshp } & \multirow[t]{2}{*}{ Rel. Freq. } & \multicolumn{3}{|c|}{ Chi2 Test } \\
\hline & & 1 & 2 & 3 & & X-sq stat & df & p-value \\
\hline \multirow[t]{4}{*}{ EcoWebVisit } & 1 & 20.3 & 14.7 & 65 & 4.5 & 12355 & 6 & $<2.20 \mathrm{E}-16$ \\
\hline & 2 & 13.2 & 19.7 & 67.1 & 8.9 & & & \\
\hline & 3 & 11.7 & 26.3 & 62 & 28.5 & & & \\
\hline & 4 & 27.2 & 33.9 & 38.9 & 58.1 & & & \\
\hline \multirow[t]{4}{*}{ BlogsVisit } & 1 & 17.2 & 16.4 & 66.4 & 6.5 & 12696 & 6 & $<2.20 \mathrm{E}-16$ \\
\hline & 2 & 11.4 & 21.8 & 66.8 & 11.8 & & & \\
\hline & 3 & 13.1 & 28.2 & 58.7 & 29.3 & & & \\
\hline & 4 & 28.4 & 33.8 & 37.8 & 52.5 & & & \\
\hline \multirow[t]{4}{*}{ BroadScWeb } & 1 & 17 & 19 & 64 & 7.6 & 13049 & 6 & $<2.20 \mathrm{E}-16$ \\
\hline & 2 & 12.5 & 24.1 & 63.4 & 15.5 & & & \\
\hline & 3 & 15.1 & 30.2 & 54.7 & 39.9 & & & \\
\hline & 4 & 32.2 & 33.5 & 34.3 & 37 & & & \\
\hline \multicolumn{2}{|l|}{ Rel. Freq. } & 21.2 & 29.6 & 49.2 & & & & \\
\hline
\end{tabular}

Source: Author's construction using the PISA 2015 Data set; ${ }^{* * *} p<0.001,{ }^{* *} p<0.01,{ }^{*} p<0.05$ 
Table 3: Chi-square test results with conditional and relative frequency distributions in \% for "IntScPrevDis"

\begin{tabular}{|c|c|c|c|c|c|c|c|c|}
\hline & & \multicolumn{3}{|c|}{ IntScPrevDis } & \multirow[t]{2}{*}{ Rel. Freq. } & \multicolumn{3}{|c|}{ Chi2 Test } \\
\hline & & 1 & 2 & 3 & & X-sq stat & $\mathrm{df}$ & p-value \\
\hline \multirow[t]{4}{*}{ EcoWebVisit } & 1 & 16.9 & 9.3 & 73.7 & 4.5 & 3097.6 & 6 & $<2.20 \mathrm{E}-16$ \\
\hline & 2 & 10.3 & 11.4 & 78.3 & 8.9 & & & \\
\hline & 3 & 6.9 & 13.2 & 79.9 & 28.5 & & & \\
\hline & 4 & 12.8 & 18.1 & 69.1 & 58.1 & & & \\
\hline \multirow[t]{4}{*}{ BlogsVisit } & 1 & 14.7 & 9.3 & 76.1 & 6.5 & 3925.7 & 6 & $<2.20 \mathrm{E}-16$ \\
\hline & 2 & 8 & 11.1 & 80.9 & 11.8 & & & \\
\hline & 3 & 7 & 13.7 & 79.3 & 29.3 & & & \\
\hline & 4 & 13.6 & 18.7 & 67.7 & 52.5 & & & \\
\hline \multirow[t]{4}{*}{ BroadScWeb } & 1 & 13.9 & 10 & 76.1 & 7.6 & 5960.5 & 6 & $<2.20 \mathrm{E}-16$ \\
\hline & 2 & 7.9 & 11.2 & 80.9 & 15.5 & & & \\
\hline & 3 & 7.1 & 14.6 & 78.3 & 39.9 & & & \\
\hline & 4 & 16.1 & 20.1 & 63.8 & 37 & & & \\
\hline \multicolumn{2}{|l|}{ Rel. Freq. } & 11.1 & & 73.2 & & & & \\
\hline
\end{tabular}

Source: Author's construction using the PISA 2015 Data set; ${ }^{* * *} p<0.001,{ }^{* *} p<0.01,{ }^{*} p<0.05$

Table 4: Posterior estimates with 95\% CI for the random country variations in students Interests

\begin{tabular}{lcl}
\hline & \multicolumn{2}{l}{ CNTRYID } \\
\cline { 2 - 3 } Dependent variables & Posterior Mean & $\mathbf{( 9 5 \%} \mathbf{C I})$ \\
\hline IntBiosph & 0.41 & $(0.20 ; 0.69)$ \\
IntScPrevDis & 0.15 & $(0.09 ; 0.23)$ \\
\hline
\end{tabular}

Note: These correspond to the point and interval estimates of the G-structure

Table 5: MCMC estimates of the residual variance-covariance matrix for the BOP model of students Interests

\begin{tabular}{lcc}
\hline & Posterior Mean & (95\% CI) \\
\hline Variance of "IntBiosph" & 2.09 & $(1.84 ; 2.40)$ \\
Covariance between "IntBiosph" and "IntScPrevDis" & 1.52 & $(1.45 ; 1.62)$ \\
Variance of "IntScPrevDis" & 1.58 & $(1.31 ; 1.81)$ \\
Correlation Coefficient & $\hat{\rho}=0.8353$ & \\
\hline
\end{tabular}

Note: This corresponds to the point and interval estimates of the $\mathrm{R}$ structure 
Table 6: MCMC estimates of the fixed effects along with their 95\% CI for the BOP model of students Interests

\begin{tabular}{|c|c|c|c|c|c|}
\hline \multirow{2}{*}{$\begin{array}{l}\text { Units } \\
\text { Fixed Effects }\end{array}$} & & \multicolumn{2}{|l|}{ IntBiosph } & \multicolumn{2}{|c|}{ IntScPrevDis } \\
\hline & & $\begin{array}{l}\text { Posterior } \\
\text { Mean }\end{array}$ & $(95 \% \mathrm{CI})$ & $\begin{array}{l}\text { Posterior } \\
\text { Mean }\end{array}$ & $(95 \% \mathrm{CI})$ \\
\hline \multicolumn{6}{|l|}{ Digital Media } \\
\hline EcoWebVisit & & -0.2 & $(-0.22 ;-0.18)$ & 0.03 & $(0.02 ; 0.05)$ \\
\hline BlogsVisit & & -0.21 & $(-0.23 ;-0.19)$ & -0.11 & $(-0.12 ;-0.09)$ \\
\hline BroadScWeb & & -0.34 & $(-0.36 ;-0.32)$ & -0.26 & $(-0.28 ;-0.24)$ \\
\hline \multicolumn{6}{|l|}{ Control Variables } \\
\hline AGE & & 0.2 & $(0.18 ; 0.22)$ & 0.18 & $(0.16 ; 0.19)$ \\
\hline Gender_Male & & -0.16 & $(-0.19 ;-0.15)$ & -0.53 & $(-0.59 ;-0.49)$ \\
\hline GradeLev & & 0.13 & $(0.11 ; 0.15)$ & 0.13 & $(0.11 ; 0.15)$ \\
\hline \multicolumn{6}{|l|}{ IMMIG } \\
\hline & Second Gen & 0.09 & $(0.05 ; 0.13)$ & 0.17 & $(0.13 ; 0.21)$ \\
\hline MISCED & & -0.03 & $(-0.03 ;-0.02)$ & -0.02 & $(-0.03 ;-0.01)$ \\
\hline FISCED & & 0.01 & $(0.001 ; 0.02)$ & 0.01 & $(0.001 ; 0.02)$ \\
\hline ESCS & & 0.16 & $(0.14 ; 0.17)$ & 0.14 & $(0.12 ; 0.16)$ \\
\hline Cut-off points & & 1.56 & $(1.50 ; 1.64)$ & 1.02 & $(0.97 ; 1.07)$ \\
\hline \multicolumn{6}{|c|}{ MCMC Algorithm specification } \\
\hline Number of Iterations & & \multicolumn{4}{|c|}{50000} \\
\hline Burn-in period & & \multicolumn{4}{|c|}{15000} \\
\hline Thinning interval & & \multicolumn{4}{|c|}{10} \\
\hline Effective Sample Size & & \multicolumn{4}{|c|}{3500} \\
\hline
\end{tabular}

